\title{
Effects of Human Development Index and Its Components on Colorectal Cancer Incidence and Mortality: a Global Ecological Study
}

\author{
Salman Khazaei ${ }^{1}$, Shahab Rezaeian ${ }^{2}$, Somayeh Khazaei ${ }^{3}$, Kamyar Mansori $^{4}$, Ali \\ Sanjari Moghaddam ${ }^{5}$, Erfan Ayubi ${ }^{6,7 *}$
}

\begin{abstract}
Geographic disparity for colorectal cancer (CRC) incidence and mortality according to the human development index (HDI) might be expected. This study aimed at quantifying the effect measure of association HDI and its components on the CRC incidence and mortality. In this ecological study, CRC incidence and mortality was obtained from GLOBOCAN, the global cancer project for 172 countries. Data were extracted about HDI 2013 for 169 countries from the World Bank report. Linear regression was constructed to measure effects of HDI and its components on CRC incidence and mortality. A positive trend between increasing HDI of countries and age-standardized rates per 100,000 of CRC incidence and mortality was observed. Among HDI components education was the strongest effect measure of association on CRC incidence and mortality, regression coefficients (95\% confidence intervals) being 2.8 (2.4, 3.2) and $0.9(0.8,1)$, respectively. HDI and its components were positively related with CRC incidence and mortality and can be considered as targets for prevention and treatment intervention or tracking geographic disparities.
\end{abstract}

Keywords: Colorectal cancer- incidence rate - mortality rate - human development index

Asian Pac J Cancer Prev, 17, Cancer Control in Western Asia Special Issue, 253-256

\section{Introduction}

Colorectal cancer (CRC) incidence and mortality rates have been well studied in developed and under developed countries. For example, an decreasing CRC incidence and mortality rate is identified in the United States and Europe (Jemal et al., 2009), however, CRC is still a public health concern in most developed countries in compared to under developed countries (Sonnenberg et al., 2000). Statistics shows an increasing trend in CRC incidence and mortality in Eastern Pacific Ocean, Middle East, African, and South American countries in recent decade, toward a similar pattern to that in the West (Sung et al., 2005).

The etiology of geographic disparity in CRC incidence and mortality can be constellation of many factors including earlier diagnosis by high effectiveness screening program, improved access to health care, or the availability of therapy modalities (Hébert et al., 2009; Altobelli et al., 2014). Socioeconomic factors have a pivotal role in embedding and quality of screening in a society or treatment tools in health care system. Previous global study showed higher CRC mortality to incidence ratio (MIR) is as a result of lower health system quality (Sunkara and Hébert, 2015). One of the important indicators for level of social and economic progress in a given country is Human Development Index (HDI). HDI is summary measure of human development, which is a composite index of three basic dimensions of human development including education as mean years of schooling, health as life expectancy at birth, and living standards as gross national income per capita.

A high quality study found that HDI is important determinant in which cancers burden such as CRC is concentrated in different regions in worldwide (Bray et al., 2012). Despite impact of human development on cancer incidence and mortality but in specific, there has been a scarcity of evidence examining how HDI related with colorectal cancer incidence and mortality, and how the relation varies by HDI components. So the objective of present study was exploration of association between HDI and colorectal incidence and mortality rate and it hypothesized that populations in low HDI regions would have a higher incidence and mortality rate in compared high HDI regions.

${ }^{1}$ Department of Biostatistics and Epidemiology, School of Public Health, Hamadan University of Medical Sciences, Hamadan, ${ }^{2}$ Social Development \& Health Promotion Research Center, Gonabad University of Medical Sciences, Gonabad, ${ }^{3}$ Rafsanjan University of Medical Sciences, Rafsanjan, ${ }^{4}$ Department of Epidemiology, School of Medicine, Kurdistan University of Medical Sciences, sanandaj, ${ }^{5}$ School of Medicine, ${ }^{6}$ School of Public Health, Shahid Beheshti University of Medical Sciences, ${ }^{7}$ Department of Epidemiology and Biostatistics, School of Public Health, Tehran University of Medical Sciences, Tehran, Iran. *For correspondence: aubi65@gmail.com 


\section{Materials and Methods}

This is an ecologic study on the relation of the age-specific incidence and mortality rate (ASR) of colorectal cancer and the HDI.

\section{Age-specific incidence rate estimate}

The methods of estimation are country-specific. The quality of the estimation depends upon the quality and information available information for each country. The methods to estimate the sex- and age-specific incidence rates of cancer for a specific country fall into one of the following broad categories: (1) rates projected to 2012 (38 countries); (2) most recent rates applied to the 2012 population (20 countries); (3) estimated from national mortality by modelling, using incidence mortality ratios derived from recorded data in country-specific cancer registries (13 countries); (4) estimated from national mortality estimates by modelling, using incidence mortality ratios derived from recorded data in local cancer registries in neighboring countries (9 European countries); (5) estimated from national mortality estimates using modeled survival (32 countries); (6) estimated as the weighted average of the local rates (16 countries); (7) one cancer registry covering part of a country is used as representative of the country profile (11 countries); (8) age/sex-specific rates for "all cancers" were partitioned using data on relative frequency of different cancers (by age and sex) (12 countries); and (9) the rates are those of neighboring countries or registries in the same area (33 countries) (Parkin, 2006; Ferlay et al., 2015).

\section{Age-specific mortality rate estimate}

Depending on the degree of detail and accuracy of the national mortality data, six methods have been utilized in the following order of priority: (1) rates projected to 2012 (69 countries); (2) most recent rates applied to the 2012 population (26 countries); (3) estimated as the weighted average of regional rates (1 country); (4) estimated from national incidence estimates by modelling, using country-specific survival (2 countries); (5) estimated from national incidence estimates using modelled survival ( 83 countries); and (6) the rates are those of neighboring countries or registries in the same area (3 countries) (Parkin, 2006; Ferlay et al., 2015). Data about the ASR for 184 countries about incidence and mortality rate of colorectal cancer for the year 2012 is obtained from the global cancer project [available at http:// globocan.iarc.
fr/Default.aspx]. GLOBOCAN also provided regional estimates for each continent.

\section{$H D I$}

Data about the HDI and other indices from 169 countries are obtained from the World Bank Report 2013 [available at http://databank.worldbank.org/data/reports. aspx]. HDI has several main components that include: life expectancy at birth, mean years of schooling, and gross national income (GNI) per capita; and also some ancillary indexes including: Percent of urbanization, and Age standardized obesity (defined as BMI $>30$ ) in adults (The weighted average of the age-specific obesity rate among adults ages 20 and older. We used the predefined categories of the distribution of HDI by country: low ( $\mathrm{HDI}<0.5)$, medium $(0.5 \leq \mathrm{HDI}<0.8)$, high $(0.8 \leq \mathrm{HDI}<0.9)$, and very high $(\mathrm{HDI} \geq 0.9)$. Therefore, these countries categorized into: Very High Human Development (27 countries), High Human Development (37 countries), Medium Human Development (89 countries) and Low Human Development (16 countries) (Bray et al., 2012). The analysis was restricted to 169 countries which both the epidemiologic data from the GLOBOCAN database and HDI were available.

\section{Statistical analysis}

Pearson coefficient was applied for assessment the correlation between HDI and CRC incidence and mortality. Analysis of variance was applied for comparisons of CRC incidence and mortality and HDI components across the categorized regions in the worlds by HDI. Linear regression models were constructed to measure the effect of HDI and its components on CRC incidence and mortality rates. Analysis was done by Stata software version 12 (StataCorp, College Station, TX, USA) by considering $\mathrm{p}$-value $<0.05$.

\section{Results}

Table 1 shows crude rate and age-standardized rates of colorectal incidence and mortality according by HDI regions. The highest age-standardized incidence and mortality rate of colorectal cancer were seen in very developed regions. More specifically, in high or very high developed regions of the world, the highest value of life expectancy at birth, mean years of schooling, gross national income per capita and total HDI was estimated to be $80.2,11.7,40$, and 0.89 respectively

Table 1. Colorectal Cancer Incidence \& Mortality and HDI Component in Different HDI Regions in 2012

\begin{tabular}{lccccccrrr}
\hline \multirow{2}{*}{ Regions } & \multicolumn{2}{c}{ CRC Incidence } & \multicolumn{2}{c}{ CRC Mortality } & \multicolumn{5}{c}{ HDI Component } \\
& CR & ASR & CR & ASR & Life Expectancy & \multicolumn{2}{c}{$\begin{array}{c}\text { Mean years } \\
\text { at Birth }\end{array}$} & $\begin{array}{c}\text { Gross national schooling } \\
\text { income per capita }\end{array}$ & HDI \\
\hline Very High HDI & 66.1 & 33.9 & 25.2 & 11.5 & 80.2 & 11.7 & $40,046.0$ & 0.9 \\
High HDI & 50.7 & 27.2 & 23.6 & 11.3 & 74.5 & 8.1 & $13,231.0$ & 0.7 \\
Medium HDI & 12.8 & 11.5 & 7.7 & 7.0 & 67.9 & 5.5 & $5,960.0$ & 0.6 \\
Low HDI & 2.7 & 4.8 & 2.1 & 3.9 & 59.4 & 4.2 & $2,904.0$ & 0.5 \\
P Value* & $<0.01$ & $<0.01$ & $<0.01$ & $<0.01$ & $<0.01$ & $<0.01$ & $<0.01$ & $<0.01$ \\
\hline
\end{tabular}

CRC, colorectal cancer; CR, Crude Rate; ASR, Age-Standardized Rates per 100,000; HDI, Human Development Index;* analysis of variance 
Table 2. Effect of HDI Components on CRC Incidence \& Mortality Rate in Linear Regression

\begin{tabular}{lcccccr}
\hline Variables & \multicolumn{3}{c}{ Incidence rate } & \multicolumn{3}{c}{ Mortality rate } \\
& $\beta$ & $\% 95 \mathrm{CI}$ & P Value & $\beta$ & $\% 95$ CI & P Value \\
\hline Life Expectancy at Birth (Year) & 0.9 & $(0.7,1)$ & $<0.01$ & 0.3 & $(0.2,0.3)$ & $<0.01$ \\
Mean years of schooling (Year) & 2.8 & $(2.4,3.2)$ & $<0.01$ & 0.9 & $(0.8,1)$ & $<0.01$ \\
& & & & & & \\
Gross national income per capita (2011 PPP \$) & 0.4 & $(0.3,0.5)$ & $<0.01$ & 0.1 & $(0.1,0.1)$ & $<0.01$ \\
Urbanization level (\%) & 0.3 & $(0.2,0.4)$ & $<0.01$ & 0.1 & $(0.1,0.1)$ & $<0.01$ \\
Age standardized obesity in adults (\%) & 0.4 & $(0.2,0.6)$ & $<0.01$ & 0.2 & $0.1,0.2)$ & $<0.01$ \\
HDI & 57.1 & $(5.3,63.8)$ & $<0.01$ & 18.3 & $(15.3,21.2)$ & $<0.01$ \\
\hline
\end{tabular}

The correlation of determination between HDI and CRC incidence and mortality were $=0.8, \mathrm{P}<0.05$ and $=0.7, \mathrm{P}<0.05$ respectively, it means the 0.9 and 0.7 of $\mathrm{CRC}$ incidence and mortality variation can be explained by HDI. The effect of HDI and its components on CRC incidence and mortality are presented in detail in Table 2. All HDI and its components positively and linearly associated with CRC incidence and mortality rates. Accordingly, increasing one unit of HDI as whole can increase the mean of CRC incidence and mortality rates by 57.1 and 18.3 respectively.

\section{Discussion}

Through application of global data, the present study demonstrated that CRC incidence and mortality more occurred in developed countries. HDI and its components associated positively with CRC incidence and mortality rates, moreover life expectancy at birth in compared other HDI components had strongest association with the CRC incidence and mortality.

Human Development Index (HDI) as a key socioeconomic determinant of health is composite of three main components including education, life expectancy, gross national income and additional factors such as urbanization level and age standardized obesity in adults. Several studies showed that some factors such as HDI components could affect the cancer incidence and mortality rates (Coughlin et al., 2007). The positive association between HDI and CRC incidence in our study was in accordance with derived evidence by Bray et al (Bray et al., 2012), who found that in medium HDI and high HDI regions an increasing trend for CRC incidence is occurred until 2008. They argued that as result of rapid societal and economic transition in medium HDI and high HDI regions registered decrease of cervical and stomach cancer might be along with increase of CRC incidence. Our finding as an extent prior knowledge shows significant effect measure of associations HDI and its components on the CRC incidence and mortality rates. Effect of HDI components on $\mathrm{CRC}$ incidence were important than CRC mortality. It might be explain by the fact that higher socioeconomic background such as income and access to knowledge foster better-informed decision and increase the health education and awareness of the risk factors for developing CRC, so people has been more motivated for participation in primary care and screening programs and the chance of CRC detection will increased (Chen et al., 2010; Crookes et al., 2014; Tavassoli et al., 2014).
The effect of income and education levels on the CRC mortality rate in our study were inconsistent with previous evidence derived individual studies so that they found educational achievements is negatively associated with CRC mortality rate (Albano et al., 2007; Menvielle et al., 2008). In other hand, it has been proved that in high income countries CRC screening programs has been embedded in the health care systems while in other regions such as Middle East countries because of some reasons including cultural and religious barriers CRC screening programs have not implemented (Arafa and Farhat, 2014).

Given that cancer incidence estimate from developing nations more registered in GLOBOCAN, one could think that the positive association between HDI components and $\mathrm{CRC}$ incidence might be more plausible and generalize to under developed and developing countries so that it was supported by some evidence from under developed and developing countries (Graham et al., 2012). In other hand, studies in developed countries found a contradict results, the CRC incidence increased with decreasing levels of neighborhood SES or educational achievement (Doubeni et al., 2012).

Apart from socioeconomic indicators on CRC incidence, an important point that should be considered in the mind is in our study the incidence of rectal cancer and colon cancer has been aggregated as CRC. The effect of SES on rectal cancer and colon cancer might be different e.g. in one longitudinal study investigators found that the effect of SES on rectal cancer in compared to colon cancer is remarkable (Brooke et al., 2016).

Using ecological datasets from GLOBOCAN and World Bank reports bring some limitation for our analysis. First, albeit national population registries embedded in GLOBOCAN dataset but they used information about cancer from smaller subnational areas or only major cities in developing nations, so GLOBOCAN data could not well generalize to all countries in the world. In other hand, it could not underline associations within countries in each HDI level regions, so our results might be followed by misinterpretation and fallacy. Second, forming a proxy for development by HDI data might be along with inherent limitations but HDI with including life expectancy measures, education indexes, gross national income and some additional components can provide a measure of development among countries and in following international comparisons of development. Third, specific associations HDI and its components on rectal cancer and colon cancer was not assessed. Finally, our results should be interpreted with caution because the ecological fallacy 
is major limitation in ecological study. In summary, based on our analysis the CRC incidence and mortality rates seem to be more concentrated in developed regions. HDI and its components positively related to $\mathrm{CRC}$ incidence and mortality.

\section{References}

Albano JD, Ward E, Jemal A, et al (2007). Cancer mortality in the United States by education level and race. J Natl Cancer Inst, 99, 1384-94.

Altobelli E, Lattanzi A, Paduano R, et al (2014). Colorectal cancer prevention in Europe: burden of disease and status of screening programs. Prev Med,62, 132-41.

Arafa M, Farhat K (2014). Colorectal cancer in the Arab world-screening practices and future prospects. Asian Pac $J$ Cancer Prev, 16, 7425-30.

Bray F, Jemal A, Grey N, et al (2012). Global cancer transitions according to the Human Development Index (2008-2030): a population-based study. Lancet Oncol, 13, 790-801.

Brooke HL, Talbäck M, Martling A, et al (2016). Socioeconomic position and incidence of colorectal cancer in the Swedish population. J Cancer Epidemiol Treat, 40, 188-95.

Chen CC, Basch CE, Yamada T (2010). An evaluation of colonoscopy use: implications for health education. J Cancer Educ, 25, 160-5.

Coughlin SS, Berkowitz Z, Hawkins NA, et al (2007). Breast and colorectal cancer screening and sources of cancer information among older women in the United States: results from the 2003 Health Information National Trends Survey. Prev Chronic Dis, 4, 57-9.

Crookes DM, Njoku O, Rodriguez MC, et al (2014). Promoting colorectal cancer screening through group education in community-based settings. J Cancer Educ, 29, 296-303.

Doubeni CA, Laiyemo AO, Major JM, et al (2012). Socioeconomic status and the risk of colorectal cancer: an analysis of over one-half million adults in the NIH-AARP Diet and Health Study. Cancer, 118, 3636-9.

Ferlay J, Soerjomataram I, Dikshit R, et al (2015). Cancer incidence and mortality worldwide: sources, methods and major patterns in GLOBOCAN 2012. Int J Cancer, 136, 359-86.

Graham A, Adeloye D, Grant L, et al (2012). Estimating the incidence of colorectal cancer in Sub-Saharan Africa: A systematic analysis. $J$ Glob Health, 2, 2040-4.

Hébert JR, Daguise VG, Hurley DM, et al (2009). Mapping cancer mortality-to-incidence ratios to illustrate racial and sex disparities in a high-risk population. Cancer, 115, 2539-52.

Jemal A, Siegel R, Ward E, et al (2009). Cancer statistics, 2009. CA Cancer J Clin, 59, 225-49.

Menvielle G, Kunst AE, Stirbu I, et al (2008). Educational differences in cancer mortality among women and men: a gender pattern that differs across Europe $B r J$ Cancer, 98, 1012-9.

Parkin DM (2006). The evolution of the population-based cancer registry. Nat Rev Cancer, 6, 603-12.

Sonnenberg A, Delcò F, Inadomi JM (2000). Cost-effectiveness of colonoscopy in screening for colorectal cancer. Ann Intern Med, 133, 573-84.

Sung JJ, Lau JY, Goh K, et al (2005). Increasing incidence of colorectal cancer in Asia: implications for screening. Lancet Oncol, 6, 871-6.

Sunkara V, Hébert JR (2015). The colorectal cancer mortality-to-incidence ratio as an indicator of global cancer screening and care. Cancer, 121, 1563-9.

Tavassoli E, Reisi M, Javadzad SH (2014). The effect of education on the improvement of fruits and vegetables consumption aiming to preventing colorectal cancer. Gastroenterol Hepatol Bed Bench, 7, 94-100. 